\title{
A millimeter-wave kinetic inductance detector camera for long-range imaging through optical obscurants
}

Sayers, Jack, Day, Peter, Cunnane, Daniel, Eom, Byeong Ho, LeDuc, Henry, et al.

Jack Sayers, Peter K. Day, Daniel P. Cunnane, Byeong Ho Eom, Henry G. LeDuc, Roger C. O'Brient, Marcus C. Runyan, Sean A. Bryan, Samuel B. Gordon, Philip D. Mauskopf, Bradley R. Johnson, Heather McCarrick, Tanay A. Bhandarkar, "A millimeter-wave kinetic inductance detector camera for long-range imaging through optical obscurants," Proc. SPIE 11411, Passive and Active Millimeter-Wave Imaging XXIII, 114110H (18 May 2020); doi: $10.1117 / 12.2557428$

Event: SPIE Defense + Commercial Sensing, 2020, Online Only, California, United States 


\title{
A Millimeter-Wave Kinetic Inductance Detector Camera for Long-Range Imaging Through Optical Obscurants
}

\author{
Jack Sayers ${ }^{\mathrm{a}}$, Peter K. Day ${ }^{\mathrm{b}}$, Daniel P. Cunnane ${ }^{\mathrm{b}}$, Byeong Ho Eom ${ }^{\mathrm{b}}$, Henry G. LeDuc ${ }^{\mathrm{b}}$, Roger \\ C. O'Brient ${ }^{b}$, Marcus C. Runyan ${ }^{b}$, Sean A. Bryan ${ }^{c}$, Samuel B. Gordon ${ }^{c}$, Philip D. Mauskopf ${ }^{c}$, \\ Bradley R. Johnson ${ }^{\mathrm{d}}$, Heather McCarrick ${ }^{\mathrm{e}}$, and Tanay A. Bhandarkarf \\ ${ }^{a}$ California Institute of Technology, Pasadena, CA 91125, USA \\ b Jet Propulsion Laboratory, Pasadena, CA 91001, USA \\ cArizona State University, Tempe, AZ 85287, USA \\ 'University of Virginia, Charlottesville, VA 22904, USA \\ ePrinceton University, Princeton, NJ 08544, USA \\ ${ }^{\mathrm{f}}$ University of Pennsylvania, Philadelphia, PA 19104, USA
}

\begin{abstract}
Millimeter-wave imaging provides a promising option for long-range target detection through optical obscurants such as fog, which often occur in marine environments. Given this motivation, we are currently developing a $150 \mathrm{GHz}$ polarization-sensitive imager using a relatively new type of superconducting pair-breaking detector, the kinetic inductance detector (KID). This imager will be paired with a $1.5 \mathrm{~m}$ telescope to obtain an angular resolution of $0.09^{\circ}$ over a $3.5^{\circ}$ field of view using 3,840 KIDs. We have fully characterized a prototype KID array, which shows excellent performance with noise strongly limited by the irreducible fluctuations from the ambient temperature background. Full-scale KID arrays are now being fabricated and characterized for a planned demonstration in a maritime environment later this year.
\end{abstract}

Keywords: Kinetic Inductance Detectors, Millimeter-Wave Imaging, Optical Obscurants

\section{INTRODUCTION}

Traditional imaging and target detection at optical wavelengths can be limited in marine environments by the presence of obscurants such as fog, clouds, rain, and snow. These obscurants are far more transparent at longer wavelengths, although diffraction can result in extremely coarse angular resolution for feasible aperture sizes. To mitigate these diffractive effects, it is therefore desirable to image at the shortest wavelengths where the atmosphere is still reasonably transparent, which corresponds to the millimeter regime. Recently, detector technologies at these wavelengths have progressed to the point where focal planes with several thousand pixels are achievable (e.g., Refs. 1,2), thus opening the possibility of high fidelity imaging at these wavelengths. As detailed below, we have adopted one of these technologies, KIDs, to develop such an imager.

\section{MILLIMETER-WAVE IMAGING THROUGH THE ATMOSPHERE}

At millimeter wavelengths, atmospheric attenuation in clear conditions is due primarily to absorption associated with internal transitions of $\mathrm{O}_{2}$ and $\mathrm{H}_{2} \mathrm{O}$ molecules. In particular, there are strong $\mathrm{O}_{2}$ absorption lines centered near $60 \mathrm{GHz}$ (e.g., Ref. 3) and $118 \mathrm{GHz}$ (e.g., Ref. 4) and $\mathrm{H}_{2} \mathrm{O}$ absorption lines centered near $183 \mathrm{GHz}$ (e.g., Ref. 5) and $325 \mathrm{GHz}$ (e.g., Ref. 6). There are atmospheric windows between these lines that are relatively transparent in most weather conditions, particular for the lower frequency windows (see Fig. 1). Within these windows, the attenuation is dominated by extremely strong Doppler-broadened $\mathrm{H}_{2} \mathrm{O}$ absorption lines above $1 \mathrm{THz}$ (Ref. 7). Various models are available to compute the atmospheric transmission for a range of different temperatures, altitudes, and humidity levels (e.g., Refs. 8-10).

Further author information: (Send correspondence to Jack Sayers)

Jack Sayers: E-mail: jack@caltech.edu, Telephone: 16263952892

Passive and Active Millimeter-Wave Imaging XXIII, edited by David A. Wikner,

Duncan A. Robertson, Proc. of SPIE Vol. 11411, 114110H · (c) 2020 SPIE

CCC code: $0277-786 \mathrm{X} / 20 / \$ 21 \cdot$ doi: $10.1117 / 12.2557428$

Proc. of SPIE Vol. $11411114110 \mathrm{H}-1$ 

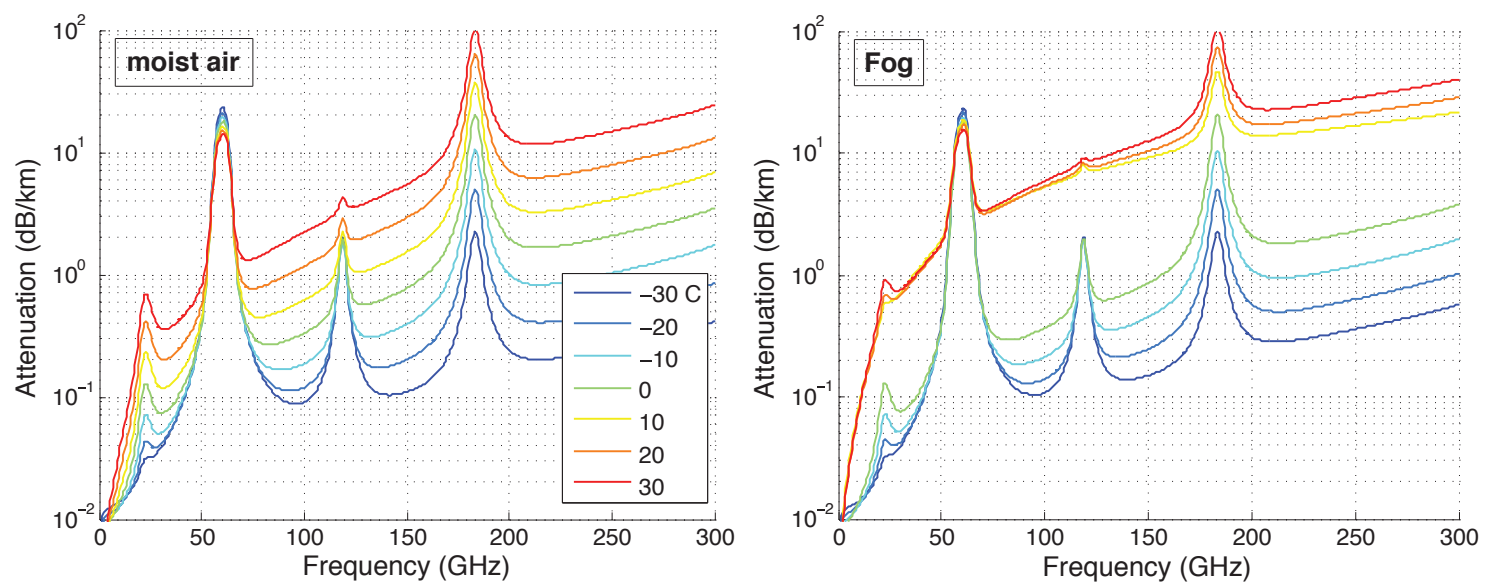

Figure 1. Atmospheric attenuation at millimeter wavelengths for saturated air (100\% relative humidity) for a range of temperatures. The left plot shows clear moist air, and the right plot shows foggy air with an optical visibility of $25 \mathrm{~m}$. For sub-freezing temperatures, there is little change in attenuation, while above freezing temperatures show only a modest increase in attenuation due to the fog. The $\mathrm{O}_{2}(60$ and $118 \mathrm{GHz})$ and $\mathrm{H}_{2} \mathrm{O}(183 \mathrm{GHz})$ absorption lines are clearly visible, along with the windows between these lines.
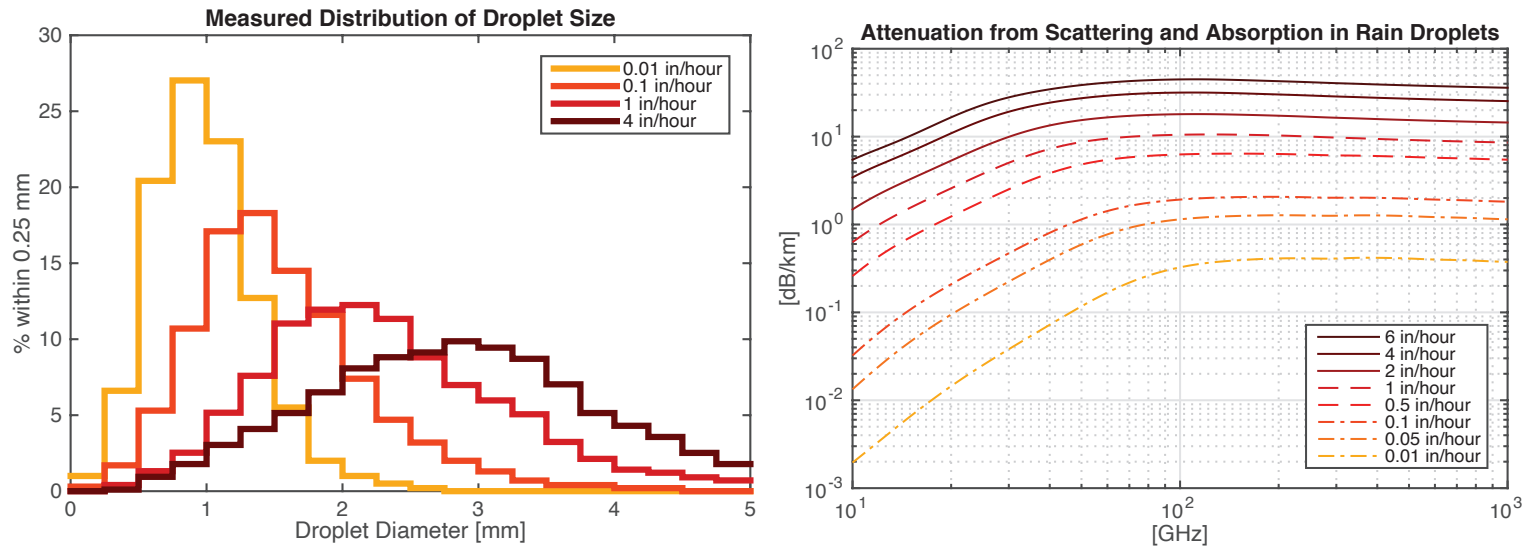

Figure 2. Left: measured distribution of rain drop sizes as a function of rainfall rate from Ref. 14. Right: calculated attenuation due to rain for a range of rainfall rates. For light to moderate rain, the attenuation is generally a few $\mathrm{dB} / \mathrm{km}$. The break in the plot shows the transition to Rayleigh scattering.

In addition to attenuation due to these molecular transitions, optical obscurants such as clouds and fog also reduce transmission at millimeter wavelengths. In above freezing conditions, these obscurants typically consist of water droplets 10s of $\mu \mathrm{m}$ in size, with ice particles occurring in below freezing conditions. Both cases can be treated using the Rayleigh absorption approximation of the Mie scattering theory from Refs. 7,8, which gives a refractive index

$$
N=\frac{3 w(\epsilon-1)}{2 m(\epsilon+2)}
$$

where $w$ is the hydrometer volume density and $m$ is the specific weight of either water or ice. The complex permittivity is given by $\epsilon$, and millimeter-wave measurements of its value are available for both water and ice (Refs. 11-13). Example calculations based on this model are given in Fig. 1, and indicate minimal attenuation in the case of ice particles and higher, but still modest, levels of attenuation in the case of water droplets.

Rain and snow present another possible mechanism for the attenuation of millimeter-wave signals. In both cases, the particle size is comparable to the wavelength (Refs. 14,15, see Fig. 2), and so the Rayleigh scattering approximation is not in general valid. For rain, the particles can be approximated as spheres following the 


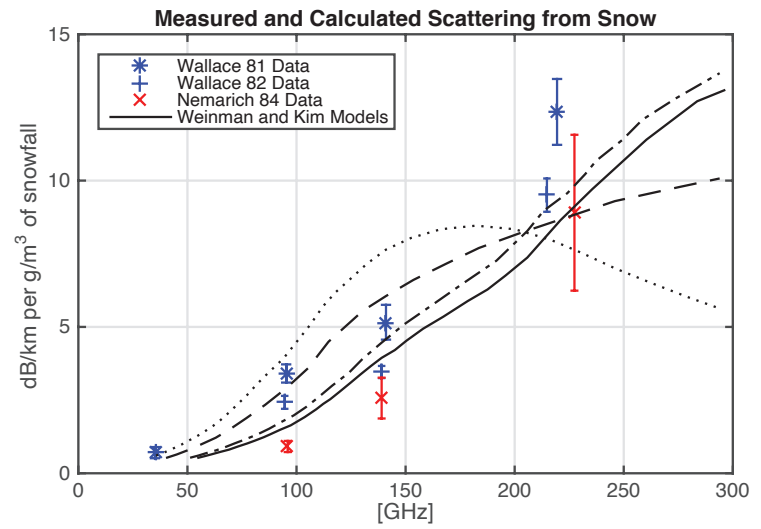

Figure 3. Calculated attenuation for a moderate snowfall rate (black lines for various assumptions of the snowflake properties), along with the measured attenuation from similar scenarios (blue and red points from Ref. 20 and Ref. 21).

rate-dependent size distributions of Ref. 14, and the number density of drops for a given rainfall rate can then be estimated from measurements of the terminal velocity of the drops (Ref. 16). An available code can be used to evaluate the Mie scattering and absorption cross sections of the drops (Ref. 17), which can then be combined with the loss tangent, dielectric constant, and rate-dependent number density of the drops to determine the total attenuation, which is shown in Fig. 2. Our calculated attenuation values are in good agreement with previous studies (e.g., Ref. 18), and the break in attenuation at millimeter wavelengths shown in the plot indicates the transition to the Rayleigh scattering regime. For moderate rainfall rates, the attenuation is a few $\mathrm{dB} / \mathrm{km}$.

For snow, there are large variations in the shape, composition, density, and size of the particles, and so approximate models such as Ref. 19 and/or measurements such as Refs. 20,21 must be used to estimate the attenuation. Fig. 3 shows the expected loss in snow, which tends to be a few $\mathrm{dB} / \mathrm{km}$ for moderate snowfall rates (similar to the attenuation in moderate rainfall). For higher (or lower) snowfall rates, the attenuation (in $\mathrm{dB}$ ) is proportional to the snowfall rate to good approximation.

\section{CONTRAST AND POLARIZATION OF MARITIME TARGETS}

The contrast between a target and its surrounding environment can be expressed as the difference in brightness temperature between the two, where

$$
T_{b}=\varepsilon T+(1-\varepsilon) T_{r}
$$

is the brightness temperature for an object with a physical temperature $T$, an emissivity $\varepsilon$, and reflected radiation with a brightness temperature $T_{r}$. In general, objects with different temperatures present a contrast, although for maritime targets the millimeter-wave contrast is often drive by differences in emissivity due to the relatively cold brightness temperature of reflected atmospheric emission at those wavelengths.

In addition, reflections from the ocean are polarized, with vertical $V$ and horizontal $H$ components given by the Fresnel equations

$$
R_{V}=\left|\frac{\epsilon_{s} \cos \theta_{i}-\sqrt{\epsilon_{s}-1+\cos ^{2} \theta_{i}}}{\epsilon_{s} \cos \theta_{i}+\sqrt{\epsilon_{s}-1+\cos ^{2} \theta_{i}}}\right|^{2}
$$

and

$$
R_{H}=\left|\frac{\cos \theta_{i}-\sqrt{\epsilon_{s}-1+\cos ^{2} \theta_{i}}}{\cos \theta_{i}+\sqrt{\epsilon_{s}-1+\cos ^{2} \theta_{i}}}\right|^{2}
$$

for incidence angle $\theta_{i}$ and sea water dielectric constant $\epsilon_{s}$. A model for $\epsilon_{s}$ valid up to $500 \mathrm{GHz}$ is given by Ref. 22 . At normal incidence, the emissivity is approximately 0.5 for both polarizations and the brightness temperature of the reflected sky is at a minimum. As the incidence angle increases, the brightness temperature of the reflected sky increases, the emissivity of the $V$ polarization increases, and the emissivity of the $H$ polarization decreases. 

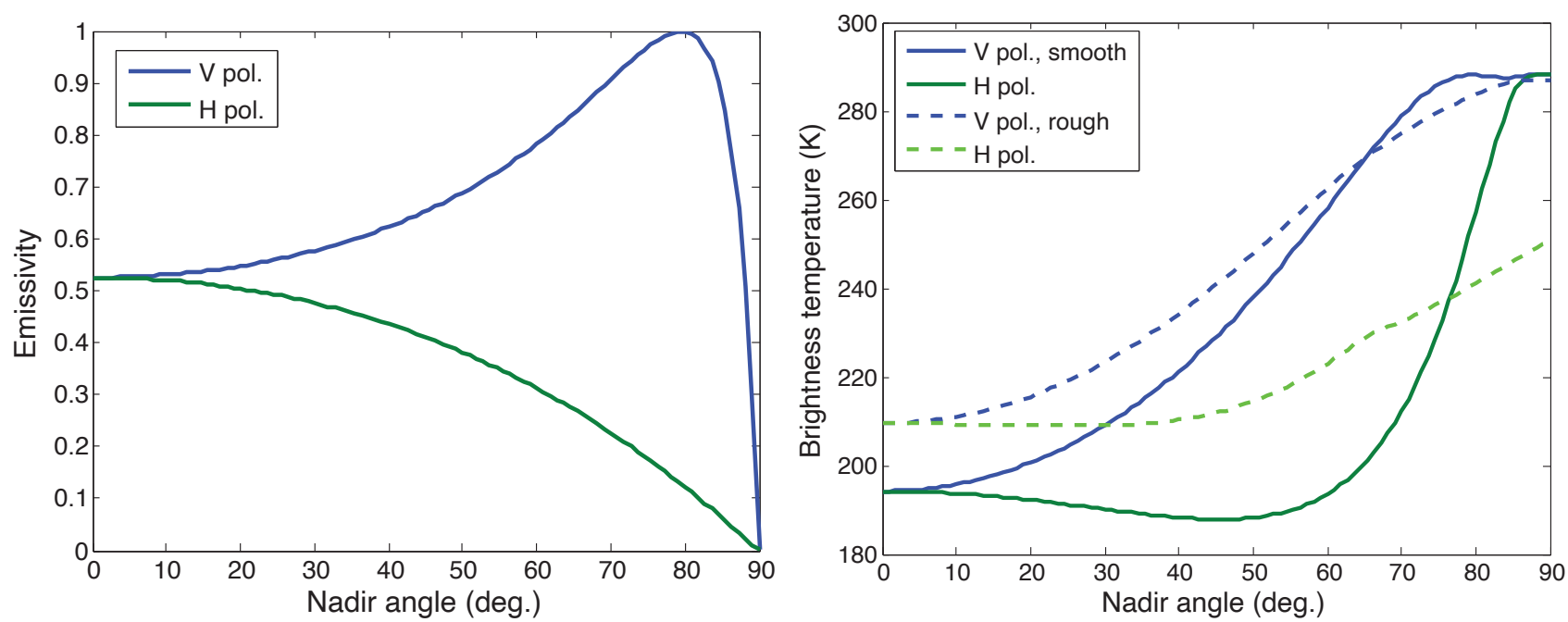

Figure 4. Left: $94 \mathrm{GHz}$ polarized emissivity of the ocean surface as a function of viewing angle. Right: $94 \mathrm{GHz}$ brightness temperature as a function of viewing angle for flat and rough ocean surfaces. The rough case corresponds to a surface wind speed of $20 \mathrm{~m} / \mathrm{s}$.

Fig. 4 shows the emissivity and effective total brightness temperature as a function of viewing angle at $94 \mathrm{GHz}$ assuming a saturated atmosphere (100\% relative humidity) at a temperature of approximately $290 \mathrm{~K}$.

Roughness of the ocean surface can be included in this calculation by considering a distribution of surface slopes. Measurements of sun glitter (Ref. 23) are consistent with a Gaussian slope distribution

$$
\rho_{s}\left(s_{x}, s_{y}\right) \sim \frac{1}{\pi g^{2}} \exp \left(-\frac{s_{x}^{2}+s_{y}^{2}}{g^{2}}\right)
$$

where $s_{x}=\delta \eta / \delta x$ and $s_{y}=\delta \eta / \delta y$ and $\eta$ is the local height. A single slope variance $g^{2}=g_{x}^{2}+g_{y}^{2}$ has been found to reasonably describe the data and to vary linearly with wind velocity $W$ according to

$$
g^{2}=0.003+0.005 W
$$

for $g$ in $\mathrm{m}$ and $W$ in $\mathrm{m} / \mathrm{s}$. The emissivity of the rough ocean surface corresponding to a particular surface wind velocity can then be estimated by integrating over this slope distribution according to

$$
\varepsilon_{r p}=\frac{\int_{-\infty}^{+\infty} d s_{x} \int_{-\infty}^{+\infty} d s_{y} \varepsilon_{r p}^{\prime} \rho_{s} w}{\int_{-\infty}^{+\infty} d s_{x} \int_{-\infty}^{+\infty} d s_{y} \rho_{s} w}
$$

where $w$ is the geometrical factor accounting for the visibility of a given surface element based on viewing angle (Ref. 24). The apparent polarized emissivities are rotated according to

$$
\varepsilon_{H}^{\prime}=\cos ^{2} \psi \varepsilon_{H}\left(\theta_{i}\right)+\sin ^{2} \psi \varepsilon_{V}\left(\theta_{i}\right)
$$

and

$$
\varepsilon_{V}^{\prime}=\sin ^{2} \psi \varepsilon_{H}\left(\theta_{i}\right)+\cos ^{2} \psi \varepsilon_{V}\left(\theta_{i}\right)
$$

where $\theta_{i}$ is the local angle of incidence and $\psi$ is the inclination of the viewing angle relative to the local coordinate system. An integral similar to Eqn. 7 can be used to average over the distribution of surface orientations. An example calculation for $W=20 \mathrm{~m} / \mathrm{s}$ is given in Fig. 4, and shows that surface roughness tends to decrease the brightness temperature of the ocean surface for viewing angles close to horizontal and increase the brightness temperature otherwise. 
Unlike the ocean itself, many potential maritime targets will have little to no polarization dependence. Depending on the construction material, the emissivity of these targets may be close to zero (e.g., aluminum) or close to unity (e.g., fiberglass). The temperature of the targets will in general be close to that of the ambient atmosphere, although modest differences are possible due to radiative and/or conductive heat transfer and heating sources associated with the target such as motors.

In order to determine the contrast of a particular target, its temperature and emissivity needs to be combined with estimates of the background brightness temperature and the transmission of the atmosphere (which can be calculated according to the procedures given above). Furthermore, the physical size of the target, combined with the ground sample distance (GSD) of the millimeter-wave imaging system, needs to be considered for any target that is not fully resolved. Specifically, for any such target the contrast will be degraded by a factor of $\sim \Delta x \Delta y / \mathrm{GSD}^{2}$, where $\Delta x$ and $\Delta y$ are the projected horizontal and vertical sizes of the target and the GSD of a millimeter-wave imager is typically limited by diffraction.

Using the above formalism, it is possible to estimate the maximum detection range for any particular target. In the presence of optical obscurants, this maximum range can often be 1-3 orders of magnitude larger in the millimeter-wave band compared to the optical band, strongly motivating the development of imagers sensitive to those wavelengths.

\section{WAKE PATTERNS}

While the target contrast detailed above is relevant for both stationary and moving targets, the latter will produce additional detectable features. In particular, a ship moving across the ocean surface will launch Kelvin wake patterns behind it (see Fig. 5). The waves locally tilt the ocean's surface, thus altering the Fresnel coefficients of the reflected radiation and generating a polarized signal that differs from the surrounding water.

The waves travel alongside the ship such that they are stationary in the ship's reference frame, forced to have a spatial wavenumber parallel to the ship's direction of travel given by

$$
k_{\|}=w / v_{\text {ship }}
$$

where $w$ is the wave's angular frequency and $v_{\text {ship }}$ is the ship's travel speed. In deep water, where the wavelength is much smaller than the depth, the waves are highly dispersive with a phase velocity $v_{\phi}=g / w$ (Ref. 25). Therefore, the wavenumber perpendicular to the ship's direction of travel is given by

$$
k_{\perp}=\sqrt{w^{4} / g^{2}-w^{2} / v_{\text {ship }}^{2}} .
$$

A comparison of $k_{\|}$and $k_{\perp}$ indicates that waves of different angular frequency travel at both different speeds and in different directions, which gives rise to the well known structure of wakes. The reciprocal of the group velocity is $\left\{\delta k_{\|} / \delta w, \delta k_{\perp} / \delta w\right\}$, and while the parallel component is constant the perpendicular component has a local minimum. Because this is a stationary point, waves with frequencies near the minimum constructively interfere to create localized wave packets. From Eqn. 11, this minimum corresponds to a spatial wavelength of

$$
\lambda=\frac{4 \pi v_{\text {ship }}^{2}}{3 g} \text {. }
$$

In order to detect the reflected signal from the wake patterns, Nyquist sampling of the spatial wavelength is required (i.e., GSD $<\lambda / 2$ ).

From Eqn. 11, these waves will propagate with a group velocity of $\sqrt{2 / 3} v_{\text {ship }}$ in a direction $\{\sqrt{2 / 3}, \sqrt{1 / 3}\}$, which corresponds to an opening angle of $\sim 35^{\circ}$ (independent of $v_{\text {ship }}$ ). Because this opening angle is universal, application of a matched spatial filter can be used to detect the feature in noisier images than would be possible for features of unknown spatial structure. These spatial templates can be constructed by summing over all angular frequencies according to

$$
\int d w f(w) \exp \left(-i\left[w\left(t-x / v_{\text {ship }}\right)-k_{\perp} y\right]\right)
$$

where $f(w)$ is the Fourier transform of the disturbance from the boat. Assuming a Gaussian distribution $f(w) \propto \exp \left(-w^{2} v_{\text {ship }} / g\right)$, an example of the resulting spatial template is given in Fig 5 . 

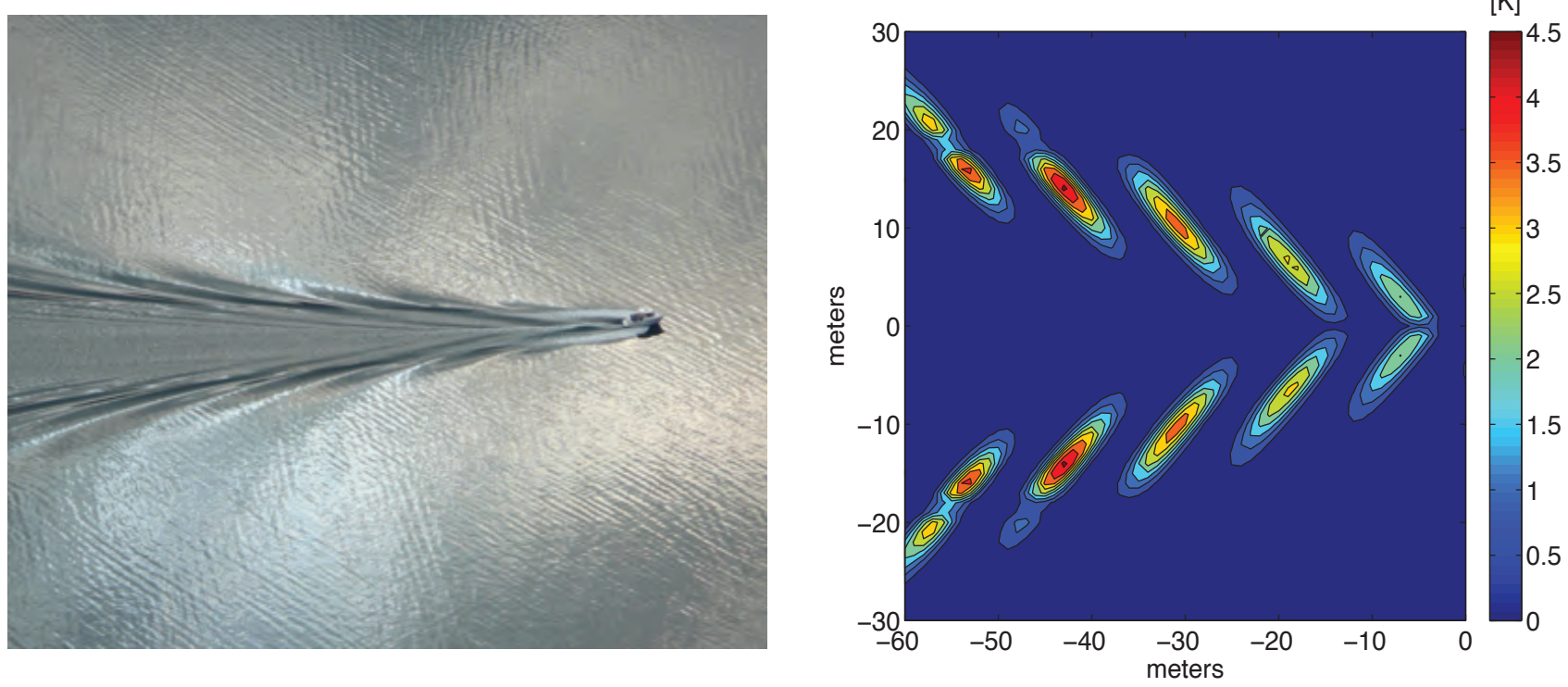

Figure 5. Left: Kelvin wake pattern produced by a ship moving on the ocean's surface as viewed from above in an airborne platform. Right: temperature contrast in polarization for the thermal radiation reflected by the wake pattern generated from a ship traveling at 12 knots triggering waves with an amplitude of $\sim 50 \mathrm{~cm}$.

\section{KIDS FOR MILLIMETER-WAVE IMAGING}

\subsection{Basic Operation}

The KID is a relatively new type of superconducting detector that operates in a non-equilibrium pair-breaking mode (e.g., Refs. 26,27). In a superconducting metal, the electrons are bound into Cooper pairs with a binding energy of $2 \Delta \simeq 3.52 k_{\mathrm{B}} T_{\mathrm{c}}$, where $T_{\mathrm{c}}$ is the superconducting transition temperature. The absorption of photons with energy $h \nu \geq 2 \Delta$ breaks the Cooper pairs into excitations called quasiparticles.

Changes in quasiparticle density $n_{\mathrm{qp}}$, such as those due to the absorption of photons, produce changes in the kinetic inductance of the superconducting film (see Fig. 6). By designing each KID to be part of a resonant circuit with a unique resonance frequency, many KIDs can be coupled to a common feedline for multiplexed readout in the frequency domain using high speed digital techniques. Multiplexing factors of $\sim 10^{3}$ have been demonstrated (e.g., Ref. 28), and are an attractive feature of KIDs compared to the other leading photometric millimeter-wave detector technology, transition edge sensors (TESs, e.g., Ref. 29).

In order to couple millimeter-wave photons to the KID, two techniques are widely in use. One method relies on an external optical-coupling element, generally a planar antenna, to collect the incident optical power and then dissipate it in the KID (e.g., Ref. 30,31). This geometry allows for some attractive design options, such as coupling multiple KIDs to a single absorbing element to, for example, define separate photometric bands. However, it generally requires the use of amorphous dielectric layers, which can contribute noise from two-level system (TLS) fluctuations. The second method is to use the KID as the optical-coupling element, allowing it to directly absorb the incident power (e.g., Ref. 32). While this geometry does not provide the same design flexibility as antenna coupling, it does simplify fabrication and eliminates the need for amorphous dielectrics.

\subsection{Detailed KID Responsivity Model}

In this section, we generally follow the formalism given in Ref. 27. For pair-breaking radiation, the quasiparticle production rate in the KID is related to the incident optical power, $P_{\mathrm{opt}}$, by

$$
\Gamma_{\mathrm{opt}}=\eta_{\mathrm{qp}} P_{\mathrm{opt}} / \Delta
$$



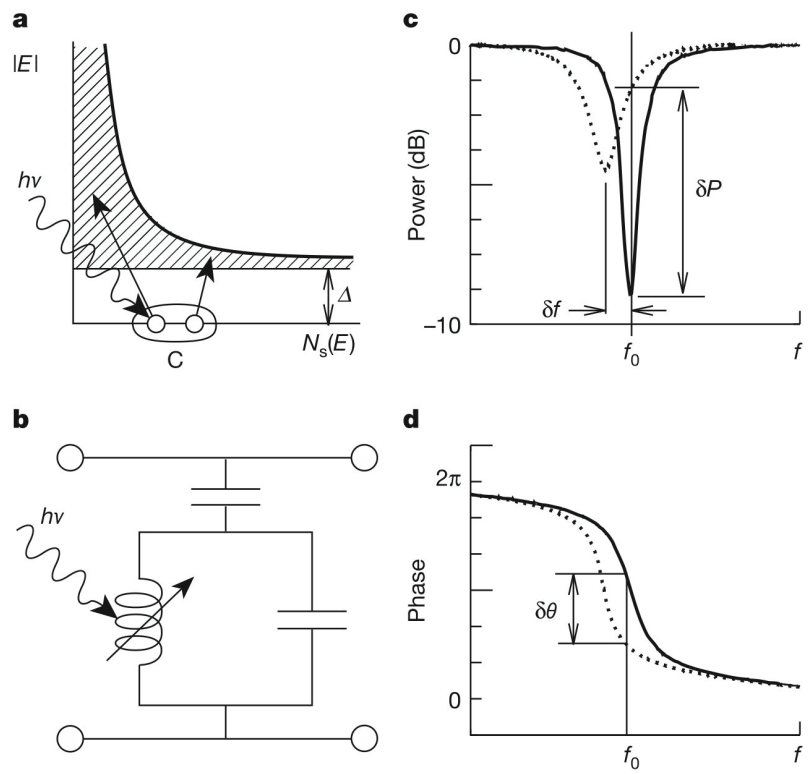
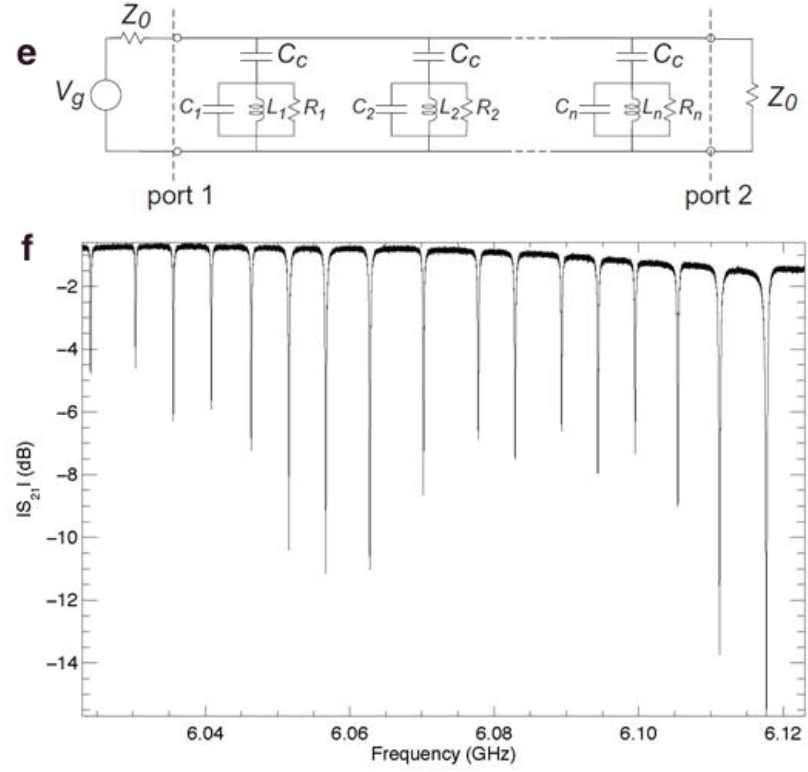

Figure 6. (a) Photons with $h \nu \geq 2 \Delta$ break Cooper pairs, creating a population of quasiparticles. (b) The increase in quasiparticle density changes the kinetic inductance of the film, which is part of a microwave resonant circuit. (c, d) As a result, the resonance frequency and quality factor of the circuit shift, changing the amplitude $(\delta P)$ and phase $(\delta \theta)$ of a carrier tone transmitted past the KID at $f_{0}$. (e) Since each KID resonant circuit can be set to a unique resonant frequency, a large number of KIDs can be connected to the same readout channel. The entire array of KIDs can thus be interrogated via a frequency comb consisting of the set of $f_{0}$ s corresponding to each resonant circuit. (f) An example of the transmission measured over a narrow frequency range for an array of KIDs, showing 17 resonances.

where $\eta_{\mathrm{qp}}$ is the efficiency with which incident power is converted into quasiparticle excitations (typically $\sim 0.6$ ). In a superconducting film of volume $V_{\mathrm{sc}}$, the recombination rate of quasiparticles into Cooper pairs is set by

$$
\Gamma_{\mathrm{R}}=\frac{n_{\mathrm{qp}} V_{\mathrm{sc}}}{\tau_{\max }}\left(1+\frac{n_{\mathrm{qp}}}{2 n^{\star}}\right)
$$

where $n_{\mathrm{qp}}$ is the density of quasiparticles, $\tau_{\max }$ corresponds to the maximum quasiparticle lifetime, and $n^{\star} \sim$ $100 \mu \mathrm{m}^{-3}$ denotes the onset of self-recombination at large quasiparticle densities (see Ref. 27). For a welldesigned detector, the number of thermally generated quasiparticles should be small compared to the number of optically generated quasiparticles, and so $\Gamma_{\mathrm{opt}}$ and $\Gamma_{\mathrm{R}}$ will be approximately equal, yielding

$$
\frac{n_{\mathrm{qp}}}{n^{\star}}=\sqrt{1+2 \frac{\eta_{\mathrm{qp}} P_{\mathrm{opt}} \tau_{\mathrm{max}}}{V_{\mathrm{sc}} \Delta n^{\star}}}-1 .
$$

In terms of $n_{\mathrm{qp}}$, the relation

$$
\tau_{\mathrm{qp}}=\frac{\tau_{\max }}{1+n_{\mathrm{qp}} / n^{\star}}
$$

has been found to provide a good description of the quasiparticle response time.

Using Mattis-Bardeen theory (Ref. 33), changes in quasiparticle density $\left(\delta n_{\mathrm{qp}}\right)$ can be converted to changes in the frequency $\left(\delta f_{\text {res }}\right)$ and dissipation $\left(\delta Q_{\text {res }}^{-1}\right)$ of the resonator. These changes produce orthogonal signals in a quadrature readout system (e.g., Ref. 34). The $\delta f_{\text {res }}$ signal is connected with the surface kinetic inductance, and generally produces a larger signal (which is often the only one considered).

In the thin film limit, the fractional change in surface impedance is given by

$$
\delta L_{\mathrm{s}} / L_{\mathrm{s}}=\kappa_{2}(\omega, T, \Delta) n_{\mathrm{qp}}
$$


where

$$
\kappa_{2}(\omega, T, \Delta)=\frac{1}{2 N_{0} \Delta}\left(1+\sqrt{\frac{2 \Delta}{\pi k_{\mathrm{B}} T}} \exp \left(-\frac{\hbar \omega}{2 k_{\mathrm{B}} T}\right) I_{0}\left(\frac{\hbar \omega}{2 k_{\mathrm{B}} T}\right)\right)
$$

and $N_{0}$ is the single spin density of states, $T$ is the temperature of the superconductor, $\omega=2 \pi f_{\text {res }}$, and $I_{0}$ is the Bessel function. A change in surface impedance can be converted to a fractional frequency shift according to

$$
\delta x=\frac{\delta f_{\text {res }}}{f_{\text {res }}}=-\frac{\alpha}{2} \frac{\delta L_{\mathrm{s}}}{L_{\mathrm{s}}}
$$

where $\alpha$ is the kinetic inductance fraction which is set by the superconducting material and the resonator geometry. For a change in optical power $\delta P_{\text {opt }}$, the fractional frequency shift is given by

$$
\mathcal{R}=\frac{\delta x}{\delta P_{\mathrm{opt}}}=-\frac{\alpha}{2} \kappa_{2}(\omega, T, \Delta) \frac{\eta_{\mathrm{qp}} \tau_{\mathrm{qp}}}{V_{\mathrm{sc}} \Delta}
$$

\subsection{Detailed KID Noise Model}

In a well designed detector, the noise should be limited by the irreducible fluctuations caused by the random arrival of photons from the target and its surroundings. For the single-moded detectors generally used at millimeter wavelengths, these fluctuations have a noise equivalent power given by

$$
N E P_{\gamma, \mathrm{amb}}^{2}=2 P_{\mathrm{amb}} h \nu_{0}\left(1+\frac{P_{\mathrm{amb}}}{N_{\mathrm{p}} h \nu_{0} \Delta \nu}\right)
$$

where $\nu_{0}$ is the millimeter-wave band center, $\Delta \nu$ is the millimeter-wave bandwidth, $P_{\text {amb }}$ is the power incident on the detector from the target and its surroundings, and $N_{\mathrm{p}}$ is the number of polarizations.

There are four main types of non-idealities in a KID imager that can potentially degrade its performance relative to the limit set by $N E P_{\gamma, \mathrm{amb}}^{2}$. As in the previous section, we largely follow the convention of Ref. 27 in describing these noise terms. The first is due to the randomness of quasiparticles recombining into Cooper pairs, with

$$
N E P_{\mathrm{REC}}^{2}=4 P_{\mathrm{opt}} \Delta / \eta_{\mathrm{qp}} \text {. }
$$

Next, the readout electronics produce noise, generally dominated by the cryogenic low-noise amplifier located immediately after the KID array in the readout chain. For a readout power $P_{\text {read }}$ at the resonator,

$$
N E P_{\mathrm{LNA}}^{2}=\frac{k_{\mathrm{B}} T_{\mathrm{N}}}{P_{\text {read }}} \frac{Q_{\mathrm{c}}^{2}}{4 Q_{\mathrm{r}}^{4} \mathcal{R}^{2}},
$$

where $T_{\mathrm{N}}$ is the noise temperature of the amplifier, $Q_{\mathrm{c}}$ is the coupling quality factor between the resonator and the readout line, $Q_{\mathrm{i}}$ is the internal quality factor of the resonator, and the total resonator quality factor is $Q_{\mathrm{r}}^{-1}=Q_{\mathrm{c}}^{-1}+Q_{\mathrm{i}}^{-1}$. In addition, there is noise due to the motion of TLSs associated with tunneling states in the dielectric material supporting the resonator. While there is no fully descriptive theory for this noise, extensive measurements have been made to determine scaling relations, with

$$
N E P_{\mathrm{TLS}}^{2}=\frac{S_{\mathrm{TLS}}}{\mathcal{R}^{2}}
$$

and $S_{\text {TLS }} \propto T^{-1.7} P_{\text {read }}^{-0.5} \mathfrak{f}^{-0.5}$ for $\mathfrak{f}$ corresponding to temporal frequency in the time-ordered data from the KID (see Refs. 34,35). Finally, non-idealities in the optical system can lead to the detector coupling to an incident optical power above what is produced by the target and its surroundings, mainly from emitting surfaces at non-zero temperature inside the cryostat and/or stray light from outside the cryostat. In this scenario, $P_{\mathrm{opt}}=P_{\mathrm{amb}}+P_{\mathrm{exc}}$, where $P_{\text {exc }}$ is the excess optical power. The total photon $N E P, N E P_{\gamma, \text { opt }}$, can thus be computed using Eqn. 22 by replacing $P_{\text {amb }}$ with $P_{\text {opt }}$.

In sum, the total noise equivalent power of a single detector will thus be

$$
N E P_{\text {tot }}^{2}=N E P_{\gamma, \text { opt }}^{2}+N E P_{\mathrm{REC}}^{2}+N E P_{\mathrm{LNA}}^{2}+N E P_{\mathrm{TLS}}^{2} \text {. }
$$



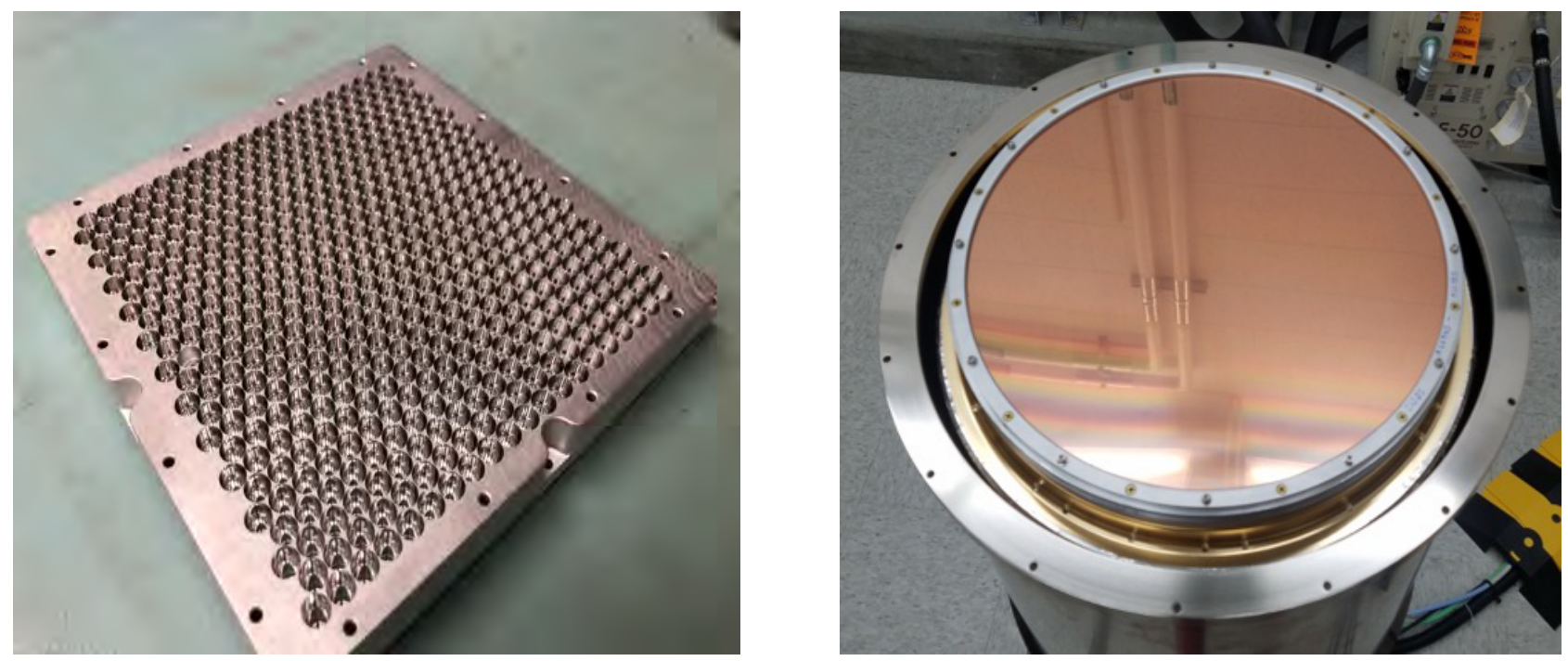

Figure 7. Left: photograph of one of the imager detector modules, with 480 feedhorns and 960 KIDs. Right: photograph of one of the metal-mesh filters installed behind the cryostat window to block out-of-band high frequency radiation.

It is possible to convert from $N E P^{2}$ (in units of $\mathrm{W}^{2} / \mathrm{Hz}$ ) to a noise equivalent temperature difference $N E T D$ (which specifies an RMS in units of $\mathrm{K}$ for a particular sampling rate $\mathcal{F}_{\mathrm{s}}$ ), with

$$
N E T D=\frac{\sqrt{N E P^{2} \mathcal{F}_{\mathrm{s}}}}{N_{\mathrm{p}} k_{\mathrm{B}} \eta_{\mathrm{opt}} \Delta \nu}
$$

where $\eta_{\text {opt }}$ is the overall optical efficiency of the imager.

\section{MILLIMETER-WAVE IMAGER CONCEPT}

\subsection{Overall Design}

We are currently developing a $150 \mathrm{GHz}$ imager using KIDs. The detector geometry is based on a feedhorncoupled design widely in use for astronomical applications at similar wavelengths (e.g., Refs. 36, 37). In this design, the waveguide section of the each feedhorn couples to two separate KIDs, each sensitive to an orthogonal linear polarization. A single hornplate contains 480 feedhorns, and couples to a single tile with 960 KIDs (see Fig. 7). The full focal plane is comprised of four such assemblies, giving a total detector count of 3,840 .

Our KID design requires operation at $\lesssim 200 \mathrm{mK}$, and we achieve these temperatures using a commercial system from STAR Cryoelectronics that uses a combination of a Gifford-McMahon (GM) cryocooler and an adiabatic demagnetization refrigerator (ADR). To accommodate the focal plane, which is approximately $205 \mathrm{~mm} \times 205 \mathrm{~mm}$ in size, a $300 \mathrm{~mm}$ diameter optical window has been installed in the cryostat. To minimize the radiative load on the GM and ADR, a series of dielectric and capacitive metal-mesh filters have been installed behind this window (Ref. 38, see Fig. 7), and the resulting hold-time at $200 \mathrm{mK}$ is approximately 10 hours.

The readout electronics for our imager are based on designs currently in use for astronomical applications (e.g., Refs. 39,40). In brief, an FPGA is used to generate a set of probe tones centered on each resonance frequency within a given tile (i.e., 960 tones). These tones are presented to an analog-to-digital converter, and then mixed up from the digital baseband to the KID baseband. The probe tones are then sent past the KID array, amplified by a cryogenic SiGe low-noise amplifier, and mixed back down to the digital baseband. An analog-to-digital converter presents the result to the FPGA, which then demodulates the signal into time-ordered data (TOD) corresponding to the transmitted amplitude and phase of each probe tone. These TOD can be collected at any desired cadence up to $\sim 10 \mathrm{kHz}$, and calibration sweeps are used to convert the amplitude and phase to the frequency shift of each resonator. Using the known position and polarization angle of each KID, these TOD can then be converted to real-time imaging data that are continuously streamed to the screen. 

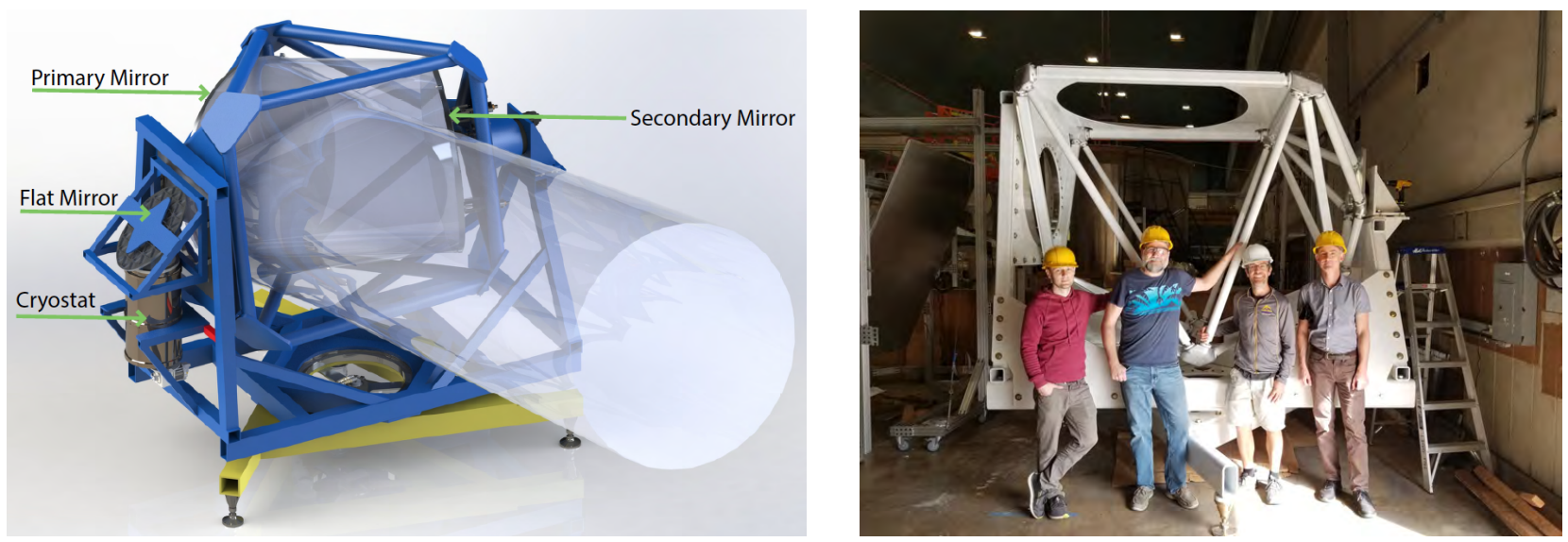

Figure 8. Left: model of the telescope with the optical path shown as a shaded cylinder and the main optical components labeled with green arrows. The mount can scan in azimuth and elevation at up to $1^{\circ} / \mathrm{s}$. Right: photograph of the assembled telescope structure with four of the authors for scale.
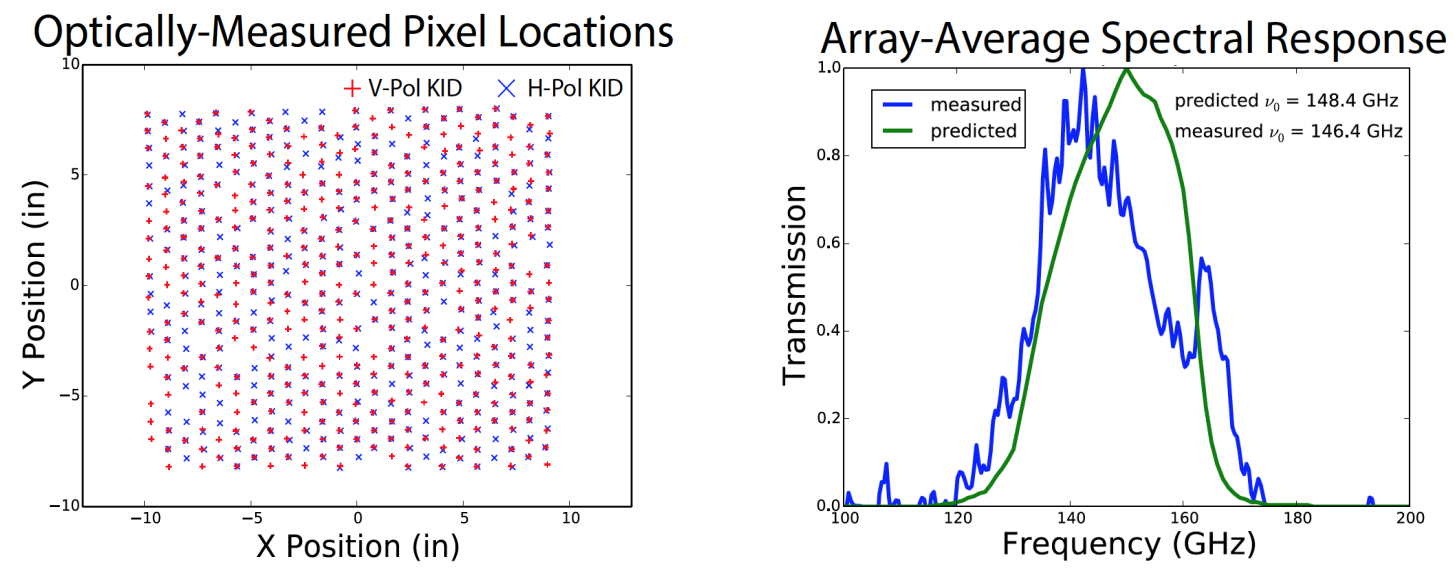

Figure 9. Left: measured locations and polarization selectivity of the detectors in one of the 960-KID arrays. Right: measured spectral response of the detectors in the 128-KID prototype array.

The imager will be paired with a telescope that has a $1.5 \mathrm{~m}$ diameter primary mirror. The optics have been designed so that the imager focal plane covers a field of view $3.5^{\circ} \times 3.5^{\circ}$ in size with a diffraction-limited angular resolution of $0.09^{\circ}$. The telescope features a crossed Dragone geometry, which provides a highly telecentric and flat focal surface to efficiently couple to the horn arrays. The Strehl ratio is $\geq 0.99$ over the entire field of view at $150 \mathrm{GHz}$. A custom az/el mount has been built for the telescope, with the ability to scan at up to $1^{\circ} / \mathrm{sec}$ with positional tolerances and deformations that negligibly impact the optical performance. The primary and secondary mirrors have been fabricated, with a surface RMS of $\simeq 50 \mu \mathrm{m}$, corresponding to $\simeq \lambda / 40$. Software to control the drive motors is currently being developed.

\subsection{Detector Characterization}

In order to validate our detector design, we performed a detailed characterization of a test array consisting of 64 feedhorns and 128 KIDs. First, we measured the position and polarization selectivity of each detector using a custom-built mapper consisting of a chopped thermal source and wire grid mounted to a X/Y translating stage and coupled to the detector array via an off-axis elliptical mirror. As expected, each detector is sensitive to a single linear polarization (either $\mathrm{H}$ or $\mathrm{V}$ ), with excellent co-alignment of the detector pairs located behind a single feedhorn and overall positions corresponding to a hexagonal close-pack array (see Fig. 9).

Next, we measured the spectral response of each detector using a custom-built Fourier transform spectrometer 
(FTS). The band edges are in good agreement with expectations, set at $\simeq 130 \mathrm{GHz}$ by the waveguide section of the feedhorn and $\simeq 170 \mathrm{GHz}$ by the series of filters placed behind to optical window. The measured spectral shape of the in-band transmission is slightly different from the prediction, although the measured band center of $146.4 \mathrm{GHz}$ is close to the expected band center of $148.4 \mathrm{GHz}$ (see Fig. 9).

In order to characterize the detector parameters, such as responsivity, optical efficiency, and excess optical loading from stray light and emission from within the cryostat, we follow the general procedure detailed in Ref. 41 including the open-source fitting software described in Ref. 42. In brief, the resonance frequency of each KID is measured over a range of detector temperatures in three different optical configurations (enclosed within a sub-K dark box, exposed to a $77 \mathrm{~K}$ thermal source outside the cryostat window, and exposed to an ambient temperature thermal source outside the cryostat window). These data are then fitted to a model described by:

$$
\begin{aligned}
& \delta x=-\frac{\alpha}{2} \kappa_{2}(\omega, T, \Delta) \times \\
& \quad\left[\left(\frac{C k_{\mathrm{B}}\left(T_{\mathrm{amb}}+T_{\mathrm{exc}}\right)}{R A_{\mathrm{sc}} \Delta}+n_{\mathrm{qp}, \mathrm{th}}^{2}\left(T_{\mathrm{qp}}, \Delta\right)+\frac{1}{R \tau_{\max }}\left(n_{\mathrm{qp}, \mathrm{th}}\left(T_{\mathrm{qp}}, \Delta\right)+\frac{1}{4 R \tau_{\max }}\right)\right)^{1 / 2}-\frac{1}{2 R \tau_{\max }}\right]
\end{aligned}
$$

where

$$
C=\frac{\eta_{\mathrm{opt}} \eta_{\mathrm{qp}} \Delta \nu}{t_{\mathrm{sc}}}
$$

includes degenerate parameters related to the conversion between incident optical power and response, $t_{\mathrm{sc}}$ is the thickness of the superconductor, $R$ is the recombination constant for the superconductor, $A_{\mathrm{sc}}$ is the surface area of the superconductor, and

$$
n_{\mathrm{qp}, \mathrm{th}}\left(T_{\mathrm{qp}}, \Delta\right)=2 N_{0} \sqrt{2 \pi k_{\mathrm{B}} T_{\mathrm{qp}} \Delta} \exp \left(-\frac{\Delta}{k_{\mathrm{B}} T_{\mathrm{qp}}}\right)
$$

is the thermal quasiparticle density for a quasiparticle temperature given by

$$
T_{\mathrm{qp}}=\left[T^{n}+\left(\frac{P_{\mathrm{opt}}}{g}\right)\right]^{1 / n},
$$

which allows for a slightly elevated quasiparticle temperature compared to the superconductor. This formalism is more general than what was presented in Sec. 5.2, and includes the effects of thermally-generated quasiparticles.

We use a two step process to fit for the free parameters in the above equations. First, we fit solely to the dark data, where $T_{\mathrm{amb}}$ and $T_{\mathrm{exc}}$ are equal to 0 and $T_{\mathrm{qp}}=T$. The value of $R$ is taken from measurements in the literature (e.g., Ref. 41), thus leaving four free parameters: the resonant frequency at infinitely low temperature $f_{\text {res }}(0)$, the kinetic inductance fraction $\alpha$, the superconductor bandgap $\Delta$, and the maximum quasiparticle lifetime $\tau_{\max }$. There is a strong degeneracy between $\alpha$ and $\Delta$, although their values can generally be tightly constrained as long as the dark data cover a sufficiently large temperature range (see Fig. 10). In our case, sweeps ranging from $75 \mathrm{mK}$ to $450 \mathrm{mK}$ were sufficient to obtain percent-level constraints, with an array median $\alpha=0.22$ and $\Delta=0.201 \mathrm{meV}$ (see Fig. 11).

The second step in the process is to fit the optical data using the posteriors on $\alpha, \Delta$, and $\tau_{\max }$ from the dark fits as priors. Because of the optical reconfiguration necessary between collecting dark data and optical data, the value of $f_{\text {res }}(0)$ generally shifts, and so its posterior from the dark fits cannot be used. Again following the general procedure of Ref. 41, we fit the frequency difference between the ambient temperature and $77 \mathrm{~K}$ optical loads, $(\delta x(\mathrm{amb})-\delta x(77 \mathrm{~K})$, see Fig. 10). The additional free parameters in this fit are the response $C$, the excess optical load $T_{\text {exc }}$, and the two parameters related to quasiparticle heating, $n$ and $\left(P_{\text {opt }} / g\right)$. We use Eqn. 29 to obtain the optical efficiency of the imager $\eta_{\text {opt }}$, assuming a value of $\eta_{\mathrm{qp}}=0.60, \Delta \nu=30 \mathrm{GHz}$, and $t_{\mathrm{sc}}=40 \pm 10 \mathrm{~nm}$. The array-median optical efficiency is 0.27 (see Fig. 11), slightly lower than the prediction of 0.38 , which includes losses in the dielectric and metal-mesh filters and the coupling between the waveguide and detector. The cause of this difference is not known, but we note that 0.27 is comparable to the best achieved optical efficiencies at these wavelengths (e.g., 0.24 for Ref. $43,0.32-0.33$ for 44 , and $0.26-0.37$ for Ref. 45). The median excess loading for the array is $41 \mathrm{~K}$ (see Fig. 11), which is small compared to the ambient load of $\simeq 300 \mathrm{~K}$ and, as detailed below, negligibly impacts the noise performance of the imager. 

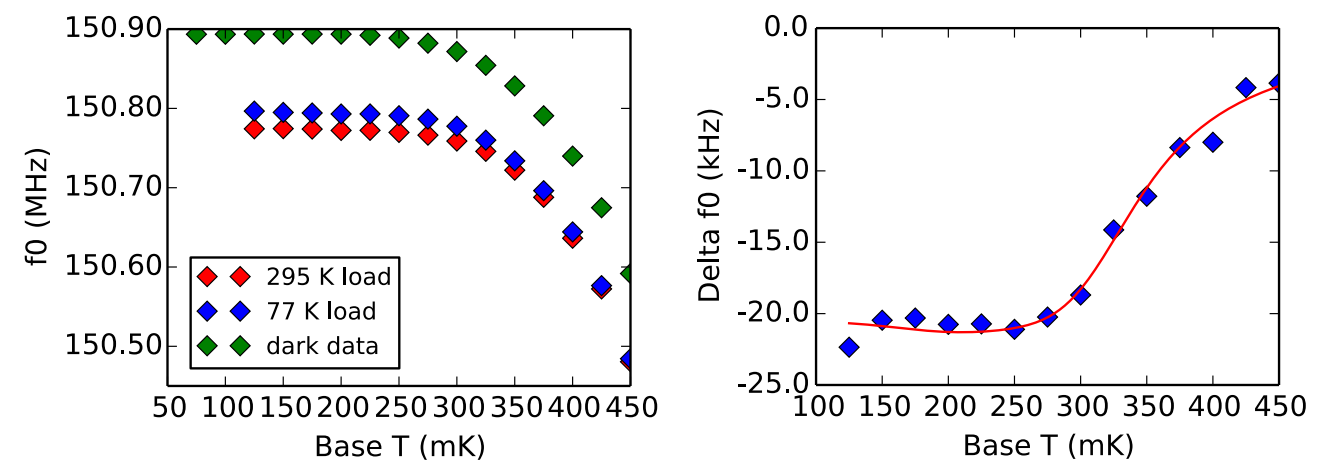

$\mathrm{C}=0.118 \pm 0.012 \mathrm{GHz} / \mathrm{nm}$

$\alpha=0.234 \pm 0.003$

$\Delta_{0}=0.202 \pm 0.000$

$T_{e x c}=14.1 \pm 18.2$

$\tau_{\max }=5.1 \pm 3.4 \mathrm{~ms}$

$n=6.4 \pm 0.8$

$\left(P_{e} / g\right)_{300 K}=227.7 \pm 209.8 \mathrm{mK}$

$A_{L}=54475 \mu \mathrm{m}^{2}$

$t_{L}=40 \pm 10 \mathrm{~nm}$

$\eta_{\text {opt }}=0.26 \pm 0.07$

$\eta_{p h}=0.60$

$\Delta \nu_{m m}=30.0 \mathrm{GHz}$
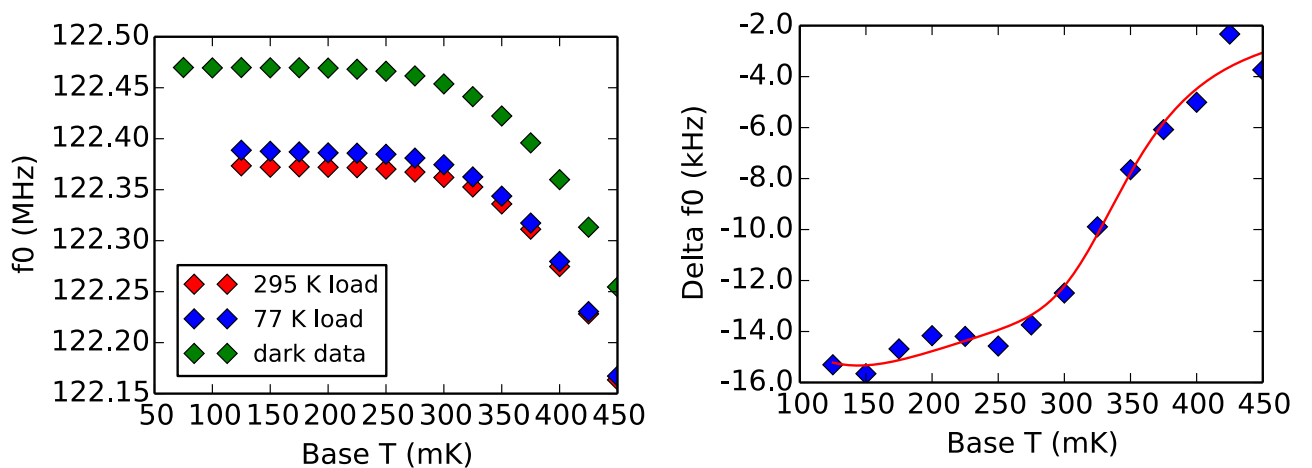

$\mathrm{C}=0.150 \pm 0.014 \mathrm{GHz} / \mathrm{nm}$

$\alpha=0.199 \pm 0.003$

$\Delta_{0}=0.200 \pm 0.001$

$T_{\text {exc }}=92.3 \pm 28.9$

$\tau_{\text {max }}=4.4 \pm 3.3 \mathrm{~ms}$

$n=4.0 \pm 0.3$

$\left(P_{e} / g\right)_{300 K}=131.7 \pm 97.3 \mathrm{mK}$

$A_{L}=54475 \mu \mathrm{m}^{2}$

$t_{L}=40 \pm 10 \mathrm{~nm}$

$\eta_{\text {opt }}=0.33 \pm 0.09$

$\eta_{p h}=0.60$

$\Delta \nu_{m m}=30.0 \mathrm{GHz}$

Figure 10. Example temperature sweeps for two resonators. Left: measured values of $f_{\text {res }}$ for three different optical configurations. Center: difference in the measured $f_{\text {res }}$ between the $295 \mathrm{~K}$ ambient temperature load and the $77 \mathrm{~K}$ load, with the best-fit model overlaid in red. Right: fitted model parameters.
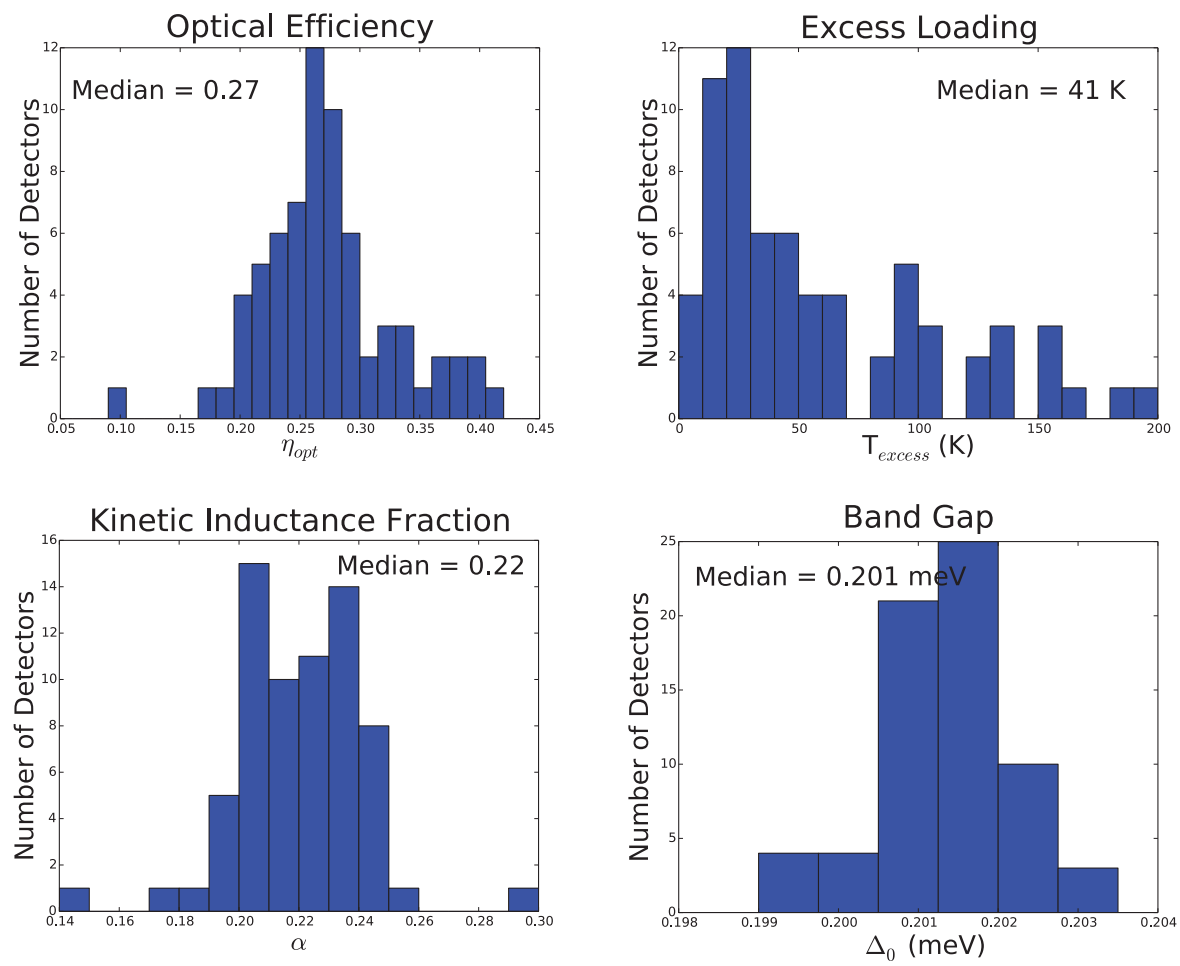

Figure 11. Histogram of fitted values for each detector in the prototype array. 

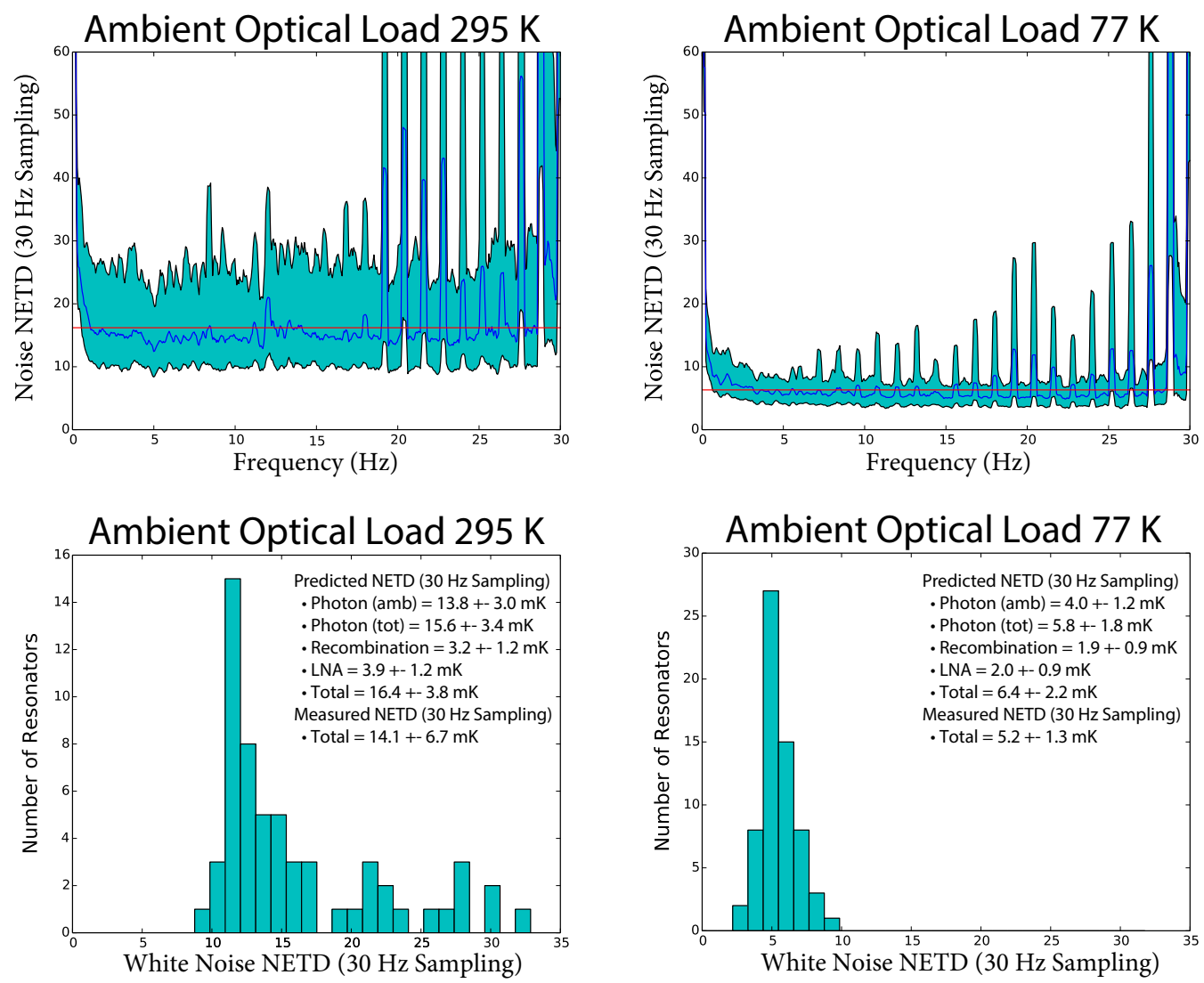

Figure 12. Top: NETD for the prototype array, with the array median in dark blue, the range enclosing $68 \%$ of the detectors in light blue, and the predicted NETD in red. Bottom: histogram of the measured white $N E T D$ for each detector with the predicted and measured $N E T D$ s shown in the legend in the upper right with the \pm values indicating the variation over the array.

In addition to performing temperature sweeps under different optical loads, we also collected TOD to characterize the noise performance of the KIDs. These data were collected at a base temperature of $200 \mathrm{mK}$ using probe tones centered on each resonance. Short frequency sweeps near each $f_{\text {res }}$ were performed to calibrate the mapping from $\delta P$ and $\delta \theta$ to $\delta f$. After converting the TOD to units of $\delta f$, the fitted resonator model described above was then used to convert from $\delta f$ to $\delta T$. Finally, noise spectra were computed from these TOD in units of $N E T D$ for a sample rate of $30 \mathrm{~Hz}$ (see Fig. 12).

The noise spectra are flat, other than discrete pickup lines associated with the GM cooler and an upturn at very low frequency due to thermal fluctuations in the optical load. There is no evidence for noise with a spectral shape corresponding to $N E T D_{\mathrm{TLS}} \sim \mathfrak{f}^{-0.25}$, which is expected for TLS noise. Therefore, we conclude that the TLS noise is negligible. The amplitude of the measured white $N E T D$ is $14.1 \pm 6.7 \mathrm{mK}$ when viewing an ambient temperature load $(295 \mathrm{~K})$ and $5.2 \pm 1.3 \mathrm{mK}$ when viewing a $77 \mathrm{~K}$ load, where the second value corresponds to the variation over the array and not to the measurement uncertainty. These values are consistent with the predicted $N E T D$ values of $16.4 \pm 3.8 \mathrm{mK}$ and $6.4 \pm 1.3 \mathrm{mK}$, where the values of $N E T D_{\gamma, \text { opt }}$ and $N E T D_{\mathrm{REC}}$ were estimated from the fitted resonator model described above and the the value of $N E T D_{\mathrm{LNA}}$ was directly measured via probe tones located away from KID resonances (see Fig. 12). Particularly for the case of an ambient temperature optical load, the total noise, $N E T D_{\text {tot }}$, is strongly dominated by the ambient photon $N E T D_{\gamma, \text { amb }}$ of $13.8 \pm 3.0 \mathrm{mK}$, indicating our detector performance is close to the ideal condition of $N E T D_{\text {tot }} \simeq N E T D_{\gamma \text {,amb. }}$. 


\subsection{Future Development Plans}

While the imager is in a highly advanced state, several tasks remain to complete it. We are currently in the process of fabricating, screening, and characterizing the full-scale 960-KID arrays. Fabrication has proven to be challenging, with some new techniques still being pursued to improve the reliability of the process. However, the arrays that do function have shown a high detector yield $(\sim 900)$. Initial characterizations of these devices indicate performance similar to what was measured for the prototype array, although with a slightly lower median optical efficiency of 0.22 and an excess loading a factor of 2-3 higher. Both of these degradations are likely due to a higher fraction of the time-reverse beam from each feedhorn being truncated by the optical opening in the cryostat, since a larger fraction of the feedhorns are close to the edges of the opening with the full-scale arrays compared to the prototype. These degradations do not significantly impact the noise performance of the imager, with the total predicted photon NETD increasing from $16.4 \mathrm{mK}$ to $18.6 \mathrm{mK}$.

The telescope frame is fully assembled, but the mirrors still need to be installed and aligned. In addition, we have developed the software to control the telescope motors, but further development is needed to enable precise scanning. Similarly, we have created a relatively basic software package for real-time imaging, but we still plan to add a range of features, such as optimal filtering, signal-to-noise estimation, user-defined refresh rates, etc.

We plan to fully test and characterize the imager at an outdoor maritime site later this year to determine how the real-world performance compares with our lab-based measurements.

\section{SUMMARY}

In the presence of optical obscurants, which often occur in marine environments, millimeter-wave photometric imaging presents an attractive option for long-range target detection. Based on this motivation, we are currently developing a $150 \mathrm{GHz}$ imager using KIDs, a relatively new type of superconducting pair-breaking detector. The imager includes 3,840 polarization-sensitive detectors, which cover a $3.5^{\circ}$ field of view with an angular resolution of $0.09^{\circ}$ when paired with the $1.5 \mathrm{~m}$ diameter telescope we have constructed. Our detailed characterization of a prototype KID array shows that the detectors perform as expected, with a noise NETD strongly limited by the irreducible fluctuations in the ambient temperature background. Full-scale detector arrays are now being fabricated and tested for a planned maritime demonstration later this year.

\section{ACKNOWLEDGMENTS}

This project has been funded by the Office of Naval Research.

\section{REFERENCES}

[1] Everett, W., Ade, P. A. R., Ahmed, Z., Anderson, A. J., Austermann, J. E., Avva, J. S., Thakur, R. B., Bender, A. N., Benson, B. A., Carlstrom, J. E., Carter, F. W., Cecil, T., Chang, C. L., Cliche, J. F., Cukierman, A., Denison, E. V., de Haan, T., Ding, J., Dobbs, M. A., Dutcher, D., Foster, A., Gannon, R. N., Gilbert, A., Groh, J. C., Halverson, N. W., Harke-Hosemann, A. H., Harrington, N. L., Henning, J. W., Hilton, G. C., Holzapfel, W. L., Huang, N., Irwin, K. D., Jeong, O. B., Jonas, M., Khaire, T., Kofman, A. M., Korman, M., Kubik, D., Kuhlmann, S., Kuo, C. L., Lee, A. T., Lowitz, A. E., Meyer, S. S., Michalik, D., Montgomery, J., Nadolski, A., Natoli, T., Nguyen, H., Noble, G. I., Novosad, V., Padin, S., Pan, Z., Pearson, J., Posada, C. M., Rahlin, A., Ruhl, J. E., Saunders, L. J., Sayre, J. T., Shirley, I., Shirokoff, E., Smecher, G., Sobrin, J. A., Stark, A. A., Story, K. T., Suzuki, A., Tang, Q. Y., Thompson, K. L., Tucker, C., Vale, L. R., Vanderlinde, K., Vieira, J. D., Wang, G., Whitehorn, N., Yefremenko, V., Yoon, K. W., and Young, M. R., "Design and Bolometer Characterization of the SPT-3G First-Year Focal Plane," Journal of Low Temperature Physics 193, 1085-1093 (12 2018).

[2] Dober, B., Austermann, J. A., Beall, J. A., Becker, D., Che, G., Cho, H. M., Devlin, M., Duff, S. M., Galitzki, N., Gao, J., Groppi, C., Hilton, G. C., Hubmayr, J., Irwin, K. D., McKenney, C. M., Li, D., Lourie, N., Mauskopf, P., Vissers, M. R., and Wang, Y., "Optical Demonstration of THz, Dual-Polarization Sensitive Microwave Kinetic Inductance Detectors," Journal of Low Temperature Physics 184, 173-179 (July 2016). 
[3] Tretyakov, M., Koshelev, M., Dorovskikh, V., Makarov, D., and Rosenkranz, P., "60-GHz oxygen band: precise broadening and central frequencies of fine-structure lines, absolute absorption profile at atmospheric pressure, and revision of mixing coefficients," Journal of Molecular Spectroscopy 231(1), 1 - 14 (2005).

[4] Koshelev, M., Delahaye, T., Serov, E., Vilkov, I., Boulet, C., and Tretyakov, M., "Accurate modeling of the diagnostic 118-GHz oxygen line for remote sensing of the atmosphere," Journal of Quantitative Spectroscopy and Radiative Transfer 196, 78 - 86 (2017).

[5] Tretyakov, M., Parshin, V., Koshelev, M., Shanin, V., Myasnikova, S., and Krupnov, A., "Studies of 183 GHz water line: Broadening and shifting by air, N2 and O2 and integral intensity measurements," Journal of Molecular Spectroscopy 218, 239-245 (04 2003).

[6] Payne, V., Mlawer, E., Cady-Pereira, K., and Moncet, J.-L., "Water vapor continuum absorption in the microwave," IEEE Transactions on Geoscience and Remote Sensing 49, 2194 - 2208 (07 2011).

[7] Liebe, H., Hufford, G., and Cotton, M., "Propagation modeling of moist air and suspended water/ice particles at frequencies below $1000 \mathrm{GHz}$," Presented at an AGARD Meeting on 'Atmospheric Propagation Effects through Natural and Man-Made Obscurants for Visible to MM-Wave Radiation,' May, 1993 (10 1993).

[8] Liebe, H., "Mpman atmospheric millimeter-wave propagation model," International Journal of Infrared and Millimeter Waves 10, 631-650 (06 1989).

[9] Paine, S., "The am atmospheric model," (09 2019).

[10] Pardo, J., Cernicharo, J., and Serabyn, E., "Atmospheric transmission at microwaves (atm): An improved model for millimeter/submillimeter applications," IEEE Transactions on Antennas and Propagation 49, 1683 - 1694 (01 2002).

[11] Ray, P. S., "Broadband complex refractive indices of ice and water," Applied Optics 11, 1836-1844 (08 1972).

[12] Warren, S. G., "Optical constants of ice from the ultraviolet to the microwave," Applied Optics 23, 12061225 (04 1984).

[13] Liebe, H., Hufford, G., and Manabe, T., "A model for the complex permittivity of water at frequencies below $1 \mathrm{THz}, "$ International Journal of Infrared and Millimeter Waves 12, 659-675 (01 1991).

[14] Laws, J. O. and Parsons, D. A., "The relation of raindrop-size to intensity," Eos, Transactions American Geophysical Union 24(2), 452-460 (1943).

[15] Locatelli, J. D. and Hobbs, P. V., "Fall speeds and masses of solid precipitation particles," Journal of Geophysical Research 79(15), 2185-2197 (1974).

[16] Foote, G. B. and Du Toit, P. S., "Terminal velocity of raindrops aloft," Journal of Applied Meteorology 8(2), 249-253 (1969).

[17] Matzler, C., "Matlab functions for mie scattering and absorption," IAP Res Rep 8 (07 2002).

[18] Zufferey, C. H., A Study of Rain Effects on Electromagnetic Waves in the 1-600 GHz Range, Master's thesis, University of Colorado (1972).

[19] Weinman, J. A. and Kim, M.-J., "A simple model of the millimeter-wave scattering parameters of randomly oriented aggregates of finite cylindrical ice hydrometeors," Journal of the Atmospheric Sciences 64(2), 634$644(2007)$.

[20] Wallace, H. B., "Millimeter-wave propagation measurements at the Ballistic Research Laboratory," IEEE Transactions on Geoscience and Remote Sensing 26, 253-258 (05 1988).

[21] Nemarich, J., Wellman, R. J., and Lacombe, J., "Backscatter and attenuation by falling snow and rain at 96, 140, and $225 \mathrm{GHz}$," IEEE Transactions on Geoscience and Remote Sensing 26, 319-329 (05 1988).

[22] Stogryn, A., "Equations for calculating the dielectric constant of saline water (correspondence)," IEEE Transactions on Microwave Theory and Techniques 19, 733-736 (08 1971).

[23] Cox, C. and Munk, W., "Measurement of the Roughness of the Sea Surface from Photographs of the Sun's Glitter," Journal of the Optical Society of America 44, 838-850 (Nov 1954).

[24] Petty, G. W., On the response of the special sensor microwave/imager to the marine environment: Implications for atmospheric parameter retrievals, PhD thesis, Washington Univ., Seattle (01 1990).

[25] Crawford, F. S., "A simple model for waterwave dispersion relations," American Journal of Physics 55(2), 171-172 (1987). 
[26] Day, P., Leduc, H., Mazin, B., Vayonakis, A., and Zmuidzinas, J., "A broadband superconducting detector suitable for use in large arrays," Nature 425, 817-21 (10 2003).

[27] Zmuidzinas, J., "Superconducting microresonators: Physics and applications," Annual Review of Condensed Matter Physics 3(1), 169-214 (2012).

[28] van Rantwijk, J., Grim, M., van Loon, D., Yates, S., Baryshev, A., and Baselmans, J., "Multiplexed readout for 1000-pixel arrays of microwave kinetic inductance detectors," IEEE Transactions on Microwave Theory and Techniques 64, 18761883 (06 2016).

[29] Irwin, K. and Hilton, G., [Transition-Edge Sensors], 63-150, Springer Berlin Heidelberg, Berlin, Heidelberg (2005).

[30] Goldin, A., Bock, J. J., Hunt, C., Lange, A. E., LeDuc, H., Vayonakis, A., and Zmuidzinas, J., "Samba: Superconducting antenna-coupled, multi-frequency, bolometric array," AIP Conference Proceedings 605(1), 251-254 (2002).

[31] Sayers, J., Bockstiegel, C., Brugger, S., Czakon, N. G., Day, P. K., Downes, T. P., Duan, R. P., Gao, J., Gill, A. K., Glenn, J., Golwala, S. R., Hollister, M. I., Lam, A., LeDuc, H. G., Maloney, P. R., Mazin, B. A., McHugh, S. G., Miller, D. A., Mroczkowski, A. K., Noroozian, O., Nguyen, H. T., Schlaerth, J. A., Siegel, S. R., Vayonakis, A., Wilson, P. R., and Zmuidzinas, J., "The status of MUSIC: the multiwavelength sub-millimeter inductance camera," in [Millimeter, Submillimeter, and Far-Infrared Detectors and Instrumentation for Astronomy VII], Holland, W. S. and Zmuidzinas, J., eds., 9153, 57 - 74, International Society for Optics and Photonics, SPIE (2014).

[32] Doyle, S., Mauskopf, P., Naylon, J., Porch, A., and Duncombe, C., "Lumped element kinetic inductance detectors," Journal of Low Temperature Physics 151(1), 530-536 (2008).

[33] Mattis, D. C. and Bardeen, J., "Theory of the anomalous skin effect in normal and superconducting metals," Physical Review 111, 412-417 (Jul 1958).

[34] Gao, J., Zmuidzinas, J., Mazin, B. A., LeDuc, H. G., and Day, P. K., "Noise properties of superconducting coplanar waveguide microwave resonators," Applied Physics Letters 90(10), 102507 (2007).

[35] Gao, J., Daal, M., Martinis, J. M., Vayonakis, A., Zmuidzinas, J., Sadoulet, B., Mazin, B. A., Day, P. K., and Leduc, H. G., "A semiempirical model for two-level system noise in superconducting microresonators," Applied Physics Letters 92(21), 212504 (2008).

[36] Dober, B. J., Ade, P. A. R., Ashton, P., Angil, F. E., Beall, J. A., Becker, D., Bradford, K. J., Che, G., Cho, H.-M., Devlin, M. J., Fissel, L. M., Fukui, Y., Galitzki, N., Gao, J., Groppi, C. E., Hillbrand, S., Hilton, G. C., Hubmayr, J., Irwin, K. D., Klein, J., Lanen, J. V., Li, D., Li, Z.-Y., Lourie, N. P., Mani, H., Martin, P. G., Mauskopf, P., Nakamura, F., Novak, G., Pappas, D. P., Pascale, E., Santos, F. P., Savini, G., Scott, D., Stanchfield, S., Ullom, J. N., Underhill, M., Vissers, M. R., and Ward-Thompson, D., "The next-generation BLASTPol experiment," in [Millimeter, Submillimeter, and Far-Infrared Detectors and Instrumentation for Astronomy VII], Holland, W. S. and Zmuidzinas, J., eds., 9153, 137 - 148, International Society for Optics and Photonics, SPIE (2014).

[37] McCarrick, H., Jones, G., Johnson, B. R., Abitbol, M. H., Ade, P. A. R., Bryan, S., Day, P., EssingerHileman, T., Flanigan, D., Leduc, H. G., and et al., "Design and performance of dual-polarization lumpedelement kinetic inductance detectors for millimeter-wave polarimetry," Astronomy \& Astrophysics 610, A45 (02 2018).

[38] Ade, P. A. R., Pisano, G., Tucker, C., and Weaver, S., "A review of metal mesh filters," in [Millimeter and Submillimeter Detectors and Instrumentation for Astronomy III], Zmuidzinas, J., Holland, W. S., Withington, S., and Duncan, W. D., eds., 6275, 248 - 262, International Society for Optics and Photonics, SPIE (2006).

[39] Duan, R., McHugh, S., Serfass, B., Mazin, B. A., Merrill, A., Golwala, S. R., Downes, T. P., Czakon, N. G., Day, P. K., Gao, J., Glenn, J., Hollister, M. I., Leduc, H. G., Maloney, P. R., Noroozian, O., Nguyen, H. T., Sayers, J., Schlaerth, J. A., Siegel, S., Vaillancourt, J. E., Vayonakis, A., Wilson, P. R., and Zmuidzinas, J., "An open-source readout for MKIDs," in [Millimeter, Submillimeter, and Far-Infrared Detectors and Instrumentation for Astronomy V], Holland, W. S. and Zmuidzinas, J., eds., 7741, 568 - 577, International Society for Optics and Photonics, SPIE (2010). 
[40] Gordon, S., Dober, B., Sinclair, A., Rowe, S., Bryan, S., Mauskopf, P., Austermann, J., Devlin, M., Dicker, S., Gao, J., and et al., "An Open Source, FPGA-Based LeKID Readout for BLAST-TNG: Pre-Flight Results," Journal of Astronomical Instrumentation 05, 1641003 (12 2016).

[41] Siegel, S. R., A Multiwavelength Study of the Intracluster Medium and the Characterization of the Multiwavelength Sub/millimeter Inductance Camera, PhD thesis, California Institute of Technology (01 2016).

[42] Carter, F. W., Khaire, T. S., Novosad, V., and Chang, C. L., "scraps: An open-source python-based analysis package for analyzing and plotting superconducting resonator data," IEEE Transactions on Applied Superconductivity 27, 1-5 (June 2017).

[43] Hui, H., Ade, P. A. R., Ahmed, Z., Alexander, K. D., Amiri, M., Barkats, D., Benton, S. J., Bischoff, C. A., Bock, J. J., Boenish, H., Bowens-Rubin, R., Buder, I., Bullock, E., Buza, V., Connors, J., Filippini, J. P., Fliescher, S., Grayson, J. A., Halpern, M., Harrison, S., Hilton, G. C., Hristov, V. V., Irwin, K. D., Kang, J., Karkare, K. S., Karpel, E., Kefeli, S., Kernasovskiy, S. A., Kovac, J. M., Kuo, C. L., Leitch, E. M., Lueker, M., Megerian, K. G., Monticue, V., Namikawa, T., Netterfield, C. B., Nguyen, H. T., O’Brient, R., IV, R. W. O., Pryke, C., Reintsema, C. D., Richter, S., Schwarz, R., Sorensen, C., Sheehy, C. D., Staniszewski, Z. K., Steinbach, B., Teply, G. P., Thompson, K. L., Tolan, J. E., Tucker, C., Turner, A. D., Vieregg, A. G., Wandui, A., Weber, A. C., Wiebe, D. V., Willmert, J., Wu, W. L. K., and Yoon, K. W., "BICEP3 focal plane design and detector performance," in [Millimeter, Submillimeter, and Far-Infrared Detectors and Instrumentation for Astronomy VIII], Holland, W. S. and Zmuidzinas, J., eds., 9914, 174 184, International Society for Optics and Photonics, SPIE (2016).

[44] Pan, Z., Ade, P. A. R., Ahmed, Z., Anderson, A. J., Austermann, J. E., Avva, J. S., Thakur, R. B., Bender, A. N., Benson, B. A., Carlstrom, J. E., Carter, F. W., Cecil, T., Chang, C. L., Cliche, J. F., Cukierman, A., Denison, E. V., de Haan, T., Ding, J., Dobbs, M. A., Dutcher, D., Everett, W., Foster, A., Gannon, R. N., Gilbert, A., Groh, J. C., Halverson, N. W., Harke-Hosemann, A. H., Harrington, N. L., Henning, J. W., Hilton, G. C., Holzapfel, W. L., Huang, N., Irwin, K. D., Jeong, O. B., Jonas, M., Khaire, T., Kofman, A. M., Korman, M., Kubik, D., Kuhlmann, S., Kuo, C. L., Lee, A. T., Lowitz, A. E., Meyer, S. S., Michalik, D., Montgomery, J., Nadolski, A., Natoli, T., Nguyen, H., Noble, G. I., Novosad, V., Padin, S., Pearson, J., Posada, C. M., Rahlin, A., Ruhl, J. E., Saunders, L. J., Sayre, J. T., Shirley, I., Shirokoff, E., Smecher, G., Sobrin, J. A., Stark, A. A., Story, K. T., Suzuki, A., Tang, Q. Y., Thompson, K. L., Tucker, C., Vale, L. R., Vanderlinde, K., Vieira, J. D., Wang, G., Whitehorn, N., Yefremenko, V., Yoon, K. W., and Young, M. R., "Optical Characterization of the SPT-3G Camera," Journal of Low Temperature Physics 193, 305-313 (Nov. 2018).

[45] Choi, S. K., Austermann, J., Beall, J. A., Crowley, K. T., Datta, R., Duff, S. M., Gallardo, P. A., Ho, S. P., Hubmayr, J., Koopman, B. J., Li, Y., Nati, F., Niemack, M. D., Page, L. A., Salatino, M., Simon, S. M., Staggs, S. T., Stevens, J., Ullom, J., and Wollack, E. J., "Characterization of the Mid-Frequency Arrays for Advanced ACTPol," Journal of Low Temperature Physics 193, 267-275 (Nov. 2018). 